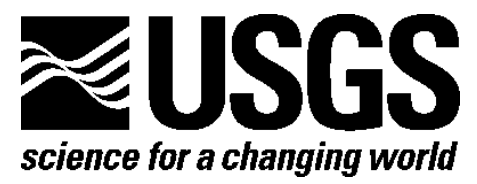

\title{
User's Manual for the Upper Delaware River Riverine Environmental Flow Decision Support System (REFDSS), Version 1.1.2
}

By Colin Talbert, Kelly O. Maloney, Chris Holmquist-Johnson, and Leanne Hanson

Open-File Report 2014-1183 


\section{U.S. Department of the Interior \\ SALLY JEWELL, Secretary}

\section{U.S. Geological Survey \\ Suzette M. Kimball, Acting Director}

U.S. Geological Survey, Reston, Virginia: 2014

For more information on the USGS-the Federal source for science about the Earth,

its natural and living resources, natural hazards, and the environment-visit

http://www.usgs.gov or call 1-888-ASK-USGS

For an overview of USGS information products, including maps, imagery, and publications, visit $h$ ttp://www.usgs.gov/pubprod

To order this and other USGS information products, visit http://store.usgs.gov

Any use of trade, firm, or product names is for descriptive purposes only and does not imply endorsement by the U.S. Government.

Although this information product, for the most part, is in the public domain, it also may contain copyrighted materials as noted in the text. Permission to reproduce copyrighted items must be secured from the copyright owner.

Suggested citation:

Talbert, Colin, Maloney, K.O., Holmquist-Johnson, C.L., and Hanson, Leanne, 2014, User's manual for the upper Delaware River riverine environmental flow decision support system (REFDSS), Version 1.1.2: U.S. Geological Survey Open-File Report 2014-1183, 23 p., http://dx.doi.org/10.3133/ofr20141183.

ISSN 2331-1258 (online) 


\section{Contents}

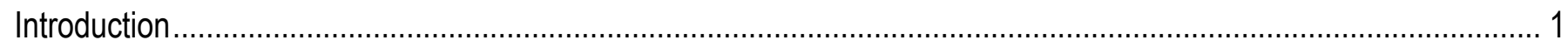

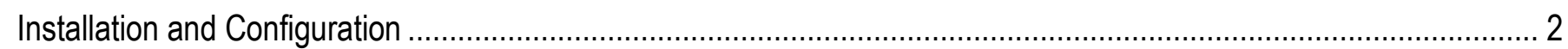

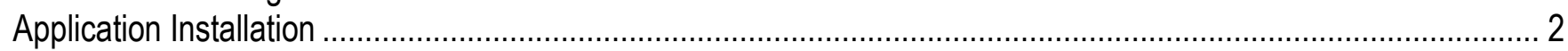

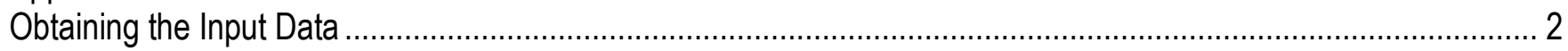

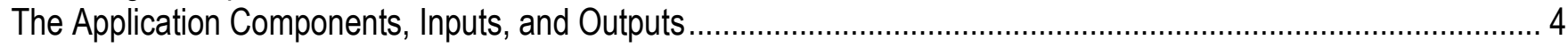

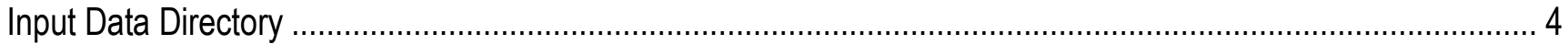

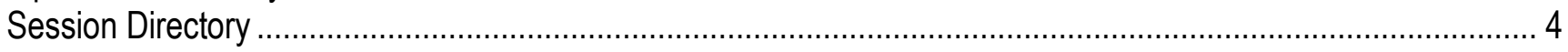

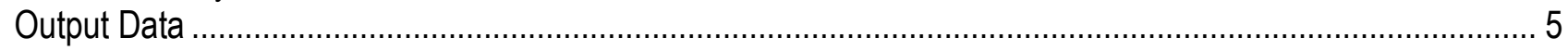

Project Configuration File (Delaware_config.xml) ............................................................................ 5

Scenarios/Flows Database (DelawareREFDSS_data.sqlite) ........................................................... 5

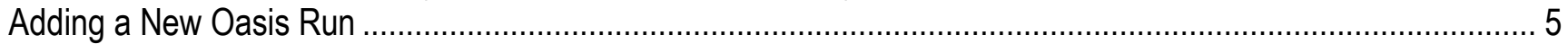

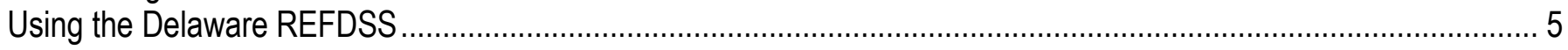

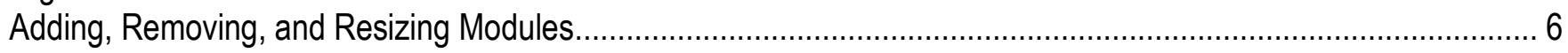

Using Views to Facilitate Organizing Multiple Modules ............................................................................. 7

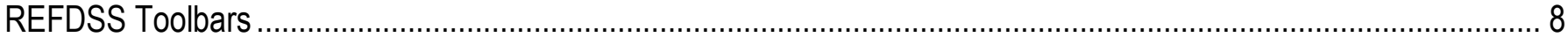

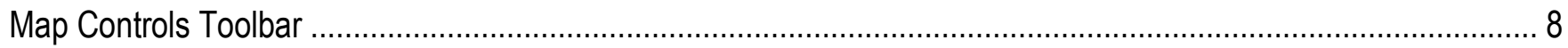

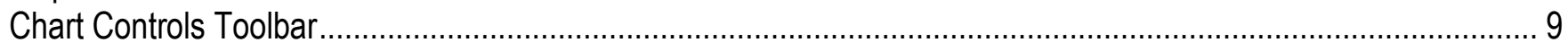

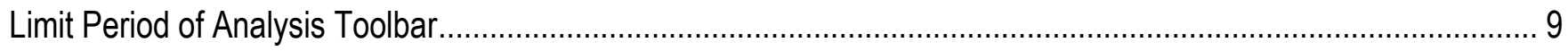

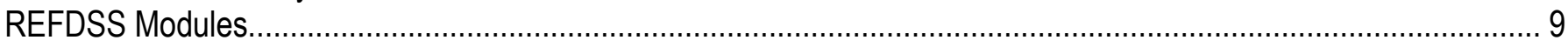

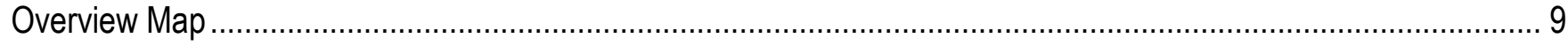

Segment Map

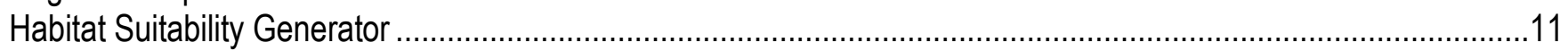

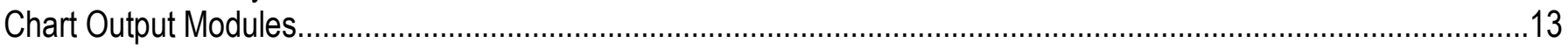

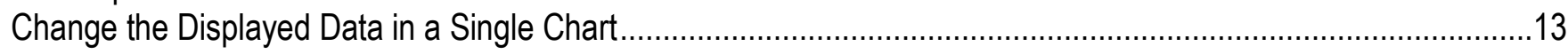

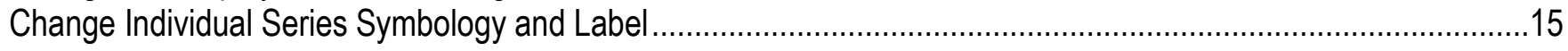

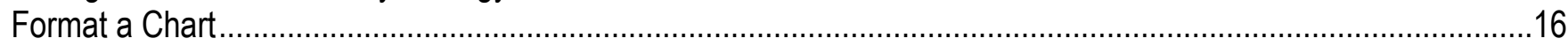

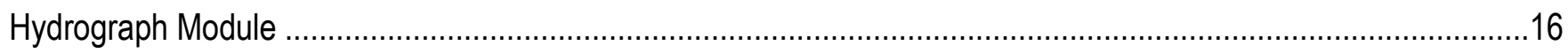

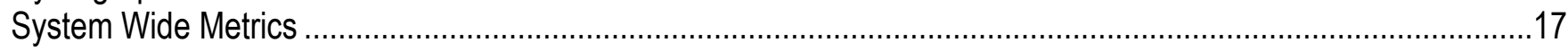

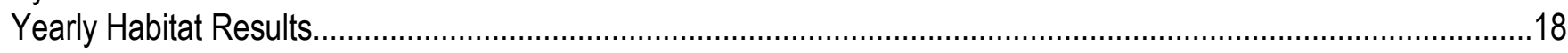

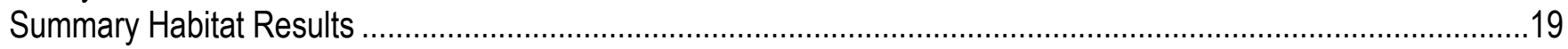

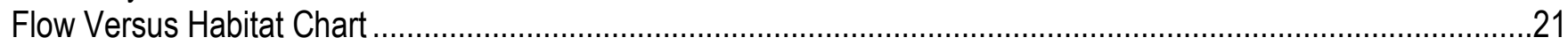

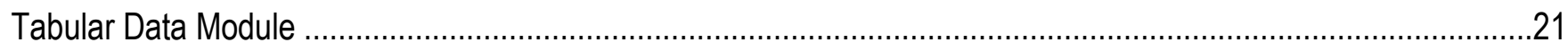

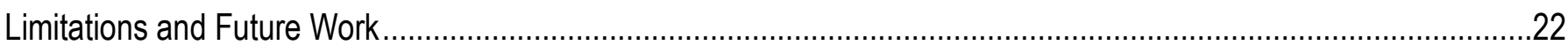

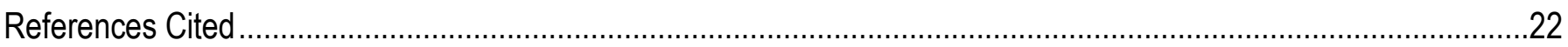

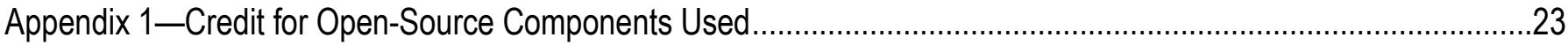

\section{Figures}

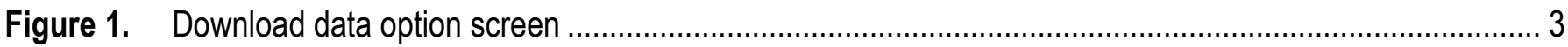

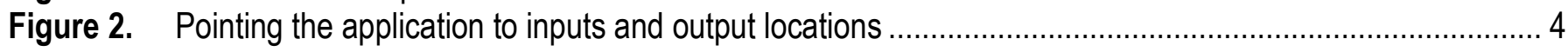

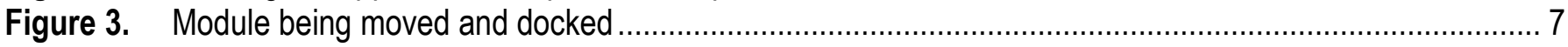

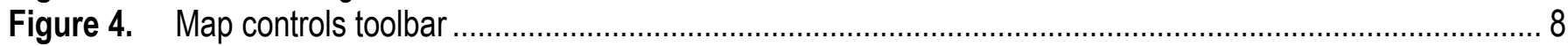




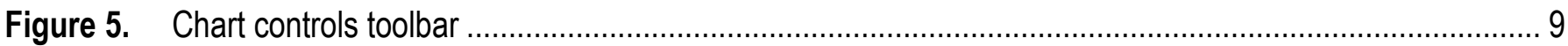

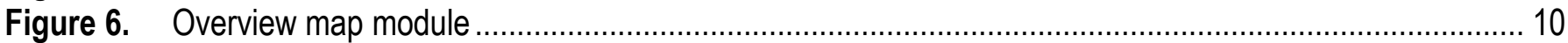

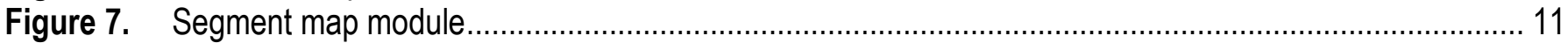

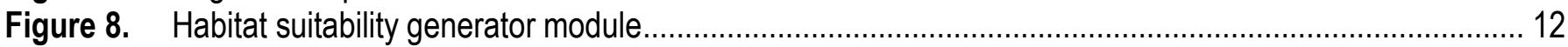

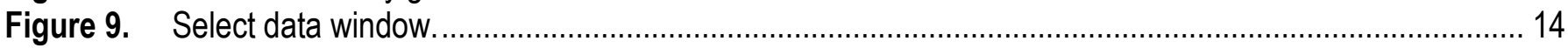

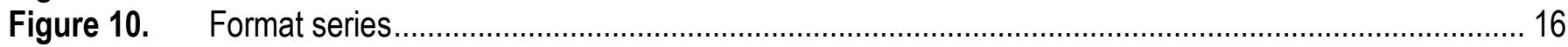

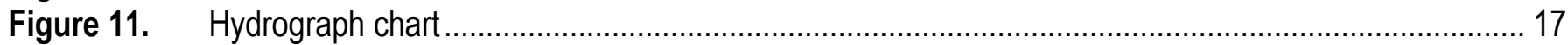

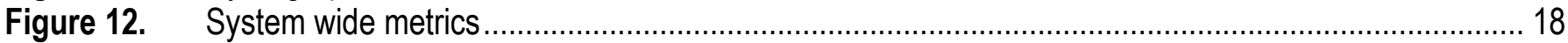

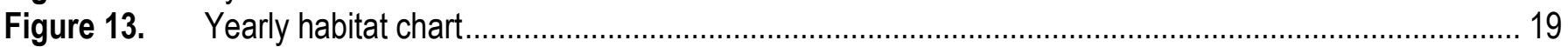

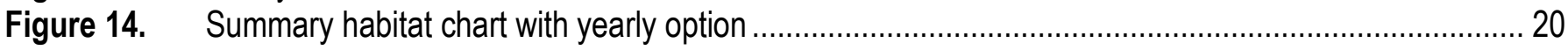

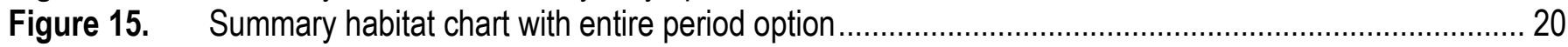

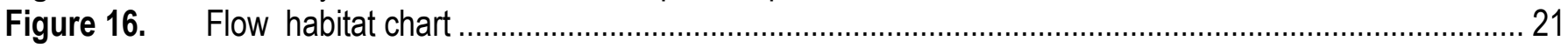

\section{Tables}

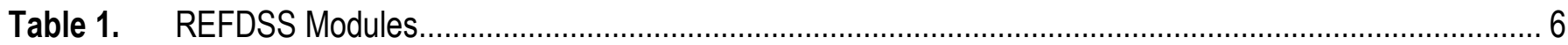

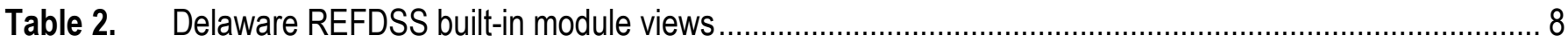




\section{User's Manual for the Upper Delaware River Riverine Environmental Flow Decision Support System (REFDSS), Version 1.1.2}

By Colin Talbert, Kelly O. Maloney, Chris Holmquist-Johnson, and Leanne Hanson

\section{Introduction}

Between 2002 and 2006, the Fort Collins Science Center (FORT) at the U.S. Geological Survey (USGS) conducted field surveys, organized workshops, and performed analysis of habitat for trout and shad in the Upper Delaware River Basin. This work culminated in the development of decision support system software (the Delaware River DSS-DRDSS, Bovee and others, 2007) that works in conjunction with the Delaware River Basin Commission's reservoir operations model, OASIS, to facilitate comparison of the habitat and water-delivery effects of alternative operating scenarios for the Basin. This original DRDSS application was developed in Microsoft Excel and is available to all interested parties through the FORT web site (http://www.fort.usgs.gov/Products/Software/DRDSS/).

Initial user feedback on the original Excel-based DSS highlighted the need for a more userfriendly and powerful interface to effectively deliver the complex data and analyses encapsulated in the DSS. In order to meet this need, the USGS FORT and Northern Appalachian Research Branch (NARB) developed an entirely new graphical user interface (GUI) application. Support for this research was through the DOI WaterSmart program (http://www.doi.gov/watersmart/html/index.php) of which the USGS component is the National Water Census (http://water.usgs.gov/watercensus/WaterSMART.html). The content and methodology of the new GUI interface emulates those of the original DSS with a few exceptions listed below. Refer to Bovee and others (2007) for the original information. Significant alterations to the original DSS include:

- We moved from Excel-based data storage and processing to a more powerful database back end powered by SQLite. The most notable effect of this is that the previous maximum temporal extent of 10 years has been replaced by a dynamic extent that can now cover the entire period of record for which we have data (1928-2000).

- We incorporated interactive geographic information system (GIS) visualization and dynamic data processing. Previous habitat maps were generated outside of the DSS in an ad hoc process that the end user could not update or investigate.

- The original bathymetric data collected in 2005 at the three main stem reaches was augmented with a higher resolution dataset collected in 2010. This new dataset was collected in order to conduct higher resolution (finer pixel size) two-dimensional (2D) hydrodynamic modeling for evaluating dwarf wedgemussel (DWM, Alasmidonta heterodon) habitat.

- Results charts are now substantially more interactive, dynamic, and accessible, which allows users to more easily focus on their particular topics of interest as well as drill down to the source data used to calculate given results. 


\section{Installation and Configuration}

\section{Application Installation}

The Delaware River REFDSS application is installed using an installer (DelawareRiver REFDSS v1 1-64bit.exe) available from the USGS ScienceBase Catalog at http://www.sciencebase.gov/DelawareREFDSS. Because of the heavy computational demands inherent in the methodology, a 64-bit computer is required. Installation requires administrative privileges. To install, simply double click the downloaded installer and follow the steps described in the installation wizard. At least 20 gigabytes (GB) of available hard drive storage space is necessary to store the inputs and derived data required by the application. This data storage space can be located on an external or network drive.

\section{Obtaining the Input Data}

The REFDSS requires a particular set of source data including:

1. Delaware_config.xml-A file used to store application and run specific settings. For example, species and "life stage" parameters.

2. DelawareREFDSS_data.sqlite_A database containing daily scenario flows and storage levels, as well as summaries of the modeled species habitat areas at various flows.

3. Inputs $\backslash$ Overview-A folder containing a series of geospatial layers used to display the study area Overview Map Module.

4. Inputs $\backslash$ Segments - A folder containing the bathymetric covariates, (environmental variables such as depth and velocity) used to derive the habitat suitability maps as well as background imagery of the study segments.

5. Outputs $\backslash$ Segments - A folder containing the derived habitat suitability maps created by the application.

Because of the size of the above data, the installer does not automatically install the required data. There are three ways to obtain these data. The first is to let the application download the data from ScienceBase as needed. This only works if the user is connected to the Internet, but it has the advantage of ensuring that the critical data organization and naming is preserved. When the application first starts, it will check that the data are available locally, and if not, the application will present the screen shown in figure 1. The user will be prompted to navigate to a folder to save the data. This workspace can be anywhere that the user has write permission. 
Welcome to the Delaware REFDSS

\section{This appears to be the first time you have opened the Delaware River Decision Support System}

If this is the first time you have opened the application, you need to obtain the required inputs.

This can be accomplished in three ways:

1) Auto download (recommended) Download Initial Data

Click here to automatically download the core data.

The application will download the required data as needed from USGS ScienceBase.

Note: You must be connected to the intemet on a reasonably fast connection for this to work.

2) Manual download from the following link (See user manual) WaterSmart on ScienceBase If there are problems with the auto download function you can manually download and unzip the oata. Click on the blue link to the right. Navigate to SourceData and download the InitialWorkspace.zip file. Unzip this to a location of your choosing.

3) Obtain the data from another user or the developer. Obtain an existing set of inputs from another user or the developer.

Click here to browse to the data location on ScienceBase.

If option 2 or 3 above is used, you will need to manual point this application to the files you obtained by following the steps below: Click'OK' on this form to open the main application.

Next click 'File' $\rightarrow$ Change Input Data Directory' on the top menu and navigate to the 'Inputs' folder.

Finally click 'File' $\rightarrow$ Open Session Directory' menu item and navigate to the 'DefaultSessionDirectoy' folder.

If you have any questions contact:

Colin Talbert

GIS Analyst and Developer

US Geological Survey

Fort Collins Science Center / North Central Climate Science Center

2150 Centre Ave. Bldg. C

Fort Collins, CO 80526

Figure 1. Download data option screen

The second method of obtaining this core data is to manually download the required data directly from ScienceBase at $h t t p: / / w w w . s c i e n c e b a s e . g o v / D e l a w a r e R E F D S S$ which can also be navigated to by clicking on the ScienceBase link on the above form (figure 1). All of the data needed by the application are in a zipped bundled called CoreWorkspace.zip which can be found by clicking the SourceData folder from the above site and then clicking on the InitialWorkspace.zip item.

The third method is to obtain the data directly from another user or the developers of the application. This might be the only option for users that do not have a reliable or high-speed bandwidth Internet connection.

None of the above methods for obtaining the required input data deliver the output geospatial layers produced by the application. However, these outputs are only required for the map displays so they will not be necessary for all users. If users are interested in viewing these outputs, they can be generated by the application using the simple process described in the section of this document describing the Habitat Suitability Generator module. 


\section{The Application Components, Inputs, and Outputs}

The Delaware REFDSS application requires several distinct data components including spatial input files, a database of daily flows and habitat areas, a location to store spatial outputs, and a configuration file used to store habitat suitability criteria (HSC) values and application settings. The names and locations of these components are critical to the application functioning correctly. While it is possible to rename, move, or edit any of these outside of the application, this is not advised for the general user. To make management of these multiple files easier for the general user, they have been organized into two groups: the "Input Data" and the "Session Directory" The location of these directories can be changed using the "File" drop-down menu (figure 2).

\begin{tabular}{|l|l|}
\hline File & Views Modules \\
Input Data \\
Change Input Data Directory \\
Session Directory \\
Open Other Session Directory \\
Save Copy of Current Session Directory as ... \\
Create New Copy of Default Session Directory \\
Add Scenario (OASIS_reformater.xls) \\
\hline Advanced Settings \\
Reset all Defaults!
\end{tabular}

Figure 2. Pointing the application to inputs and output locations

\section{Input Data Directory}

The first component of the REFDSS is the "Input Data Directory" folder. This folder contains the map inputs used by the application for display and geoprocessing. The main folder contains two subfolders, one for the overview map layers and one for the other individual layers for each river segment (reach). All of the input files are static and do not change regardless of subsequent processing. Unzipped, this component is about 10 gigabytes (GB) in size, so if users plan to run the application with multiple session folders, it would be most efficient for each to point to the same set of inputs. This is the default behavior in the application.

\section{Session Directory}

The second component is the "Session Directory." This folder contains the remainder of the data components organized into a single directory. This folder can be thought of as an individual "run" or project workspace. Users that want to compare outputs from different HSCs or Oasis models will need to create multiple session directories, one for each run. This can be easily done by selecting the "Save Copy of Current Directory as [...]" or the "Create New Copy of Default Session Directory" options from the "File" menu. Each of these different session directories can have unique HSCs or collections of OASIS inputs. Switching between these is done with the "Open Other Session Directory" option. 
Most users will not need to interact directly with the individual data components that make up a session directory, but they are described in the following sections. They can be specified individually by using the "Advanced Settings" option, but this is neither recommended nor supported for most users.

\section{Output Data}

This subfolder contains the GIS output maps generated using the "Edit habitat suitability curves/maps" view. It contains an additional 7 GB of data, so for efficiency, when you first install the application, these layers are not provided. These layers are only used for display in the individual segment map modules. The data used to drive the chart and tabular output have already been extracted and stored separately. If you are not interested in viewing the output maps, then you do not need to generate these maps to view the output charts. If you are interested in viewing the output GIS maps, you will need to first regenerate these layers by going to the "Edit habitat suitability curves/maps" view and clicking the "Process all species" button. Note: doing so would result in the REFDSS recalculating all geospatial model outputs which can be time consuming. Switching views is described in the "Using views to facilitate organizing multiple modules" section of this document."

Project Configuration File (Delaware_config.xml)

The project configuration file stores all the individual pieces of information used by the application. This includes many items that are specific to an individual user such as changes to parameters, hydroperiods, or HSC. This file also contains the history of all changes you've made to charts, symbology, and saved views, among others. Note that default values to this file can be restored at any point by clicking on "File" and selecting "Reset all Defaults!"

\section{Scenarios/Flows Database (DelawareREFDSS_data.sqlite)}

This is the database that contains all of the flow and other daily values from each flow management scenario. Additionally, it contains summary statistics extracted from the habitat map output generated by the application.

\section{Adding a New Oasis Run}

The Upper Delaware River REFDSS includes three flow management scenarios: Rev1, Rev7, and the flexible flow management plan (ffmp) provided by the Delaware River Basin Commission (Hernan Quinodoz, DRBC, personal comm., July 6, 2012). The first two were included in the original DSS although the versions we are using here are more current than the versions used in the original DSS. The FFMP was developed after the original DSS. To import a new OASIS scenario, you must first use the Excel-based reformatter created for the original DSS which is provided in the inputs folder used by the REFDSS (for example: ...Inputs $\backslash$ OASIS reformatterV2_1_ffmp.xls). Instructions for its use are in cells A2-A4 on the "ToDSS" page of the workbook. After following the update instructions, saving, and closing Excel, you can import the scenario by clicking the "Add Scenario (OASIS_reformatter.xls)" button from the "File" menu. Be aware that the code to import a new scenario has not yet been optimized so the process can take up to 15 minutes. Future versions of the REFDSS might include tools for batch importing of multiple OASIS runs.

\section{Using the Delaware REFDSS}

The REFDSS application is set up in a modular manner to allow flexibility in the content displayed at any time. Currently, there are nine different modules in the application (table 1). Each 
module consists of an independent window configured for viewing a specific type of data or summary. When using the application, any number of modules can be used at any time. Each module is independently configurable, resizable, and moveable. While each open module is independent, they do interact to facilitate data analysis. For example, moving the cursor on a hydrograph and clicking once will update the maps to display the selected flow. If the user does not want an individual module updated relative to other modules, the user can lock individual modules by right clicking anywhere on the module and selecting "Lock." Locked modules are denoted with an asterisk $(*)$ after their name. Each module will be described in detail in subsequent sections.

Table 1. REFDSS modules.

\begin{tabular}{|c|c|}
\hline Module & Description \\
\hline Overview Map & GIS overview map showing location of each river reach \\
\hline Segment Map & GIS map of a single river reach \\
\hline $\begin{array}{l}\text { Habitat Suitability } \\
\text { Generator }\end{array}$ & $\begin{array}{l}\text { Used to view and modify the habitat suitability criteria used in the DSS. Also used to regenerate the } \\
\text { spatial habitat map output }\end{array}$ \\
\hline Hydrograph & Chart of the daily flow, habitat, or other metrics \\
\hline $\begin{array}{l}\text { System Wide } \\
\text { Metrics }\end{array}$ & Chart of non-habitat metrics (e.g., drought days) summarized over the period of record \\
\hline $\begin{array}{l}\text { Yearly Habitat } \\
\text { Results }\end{array}$ & Chart of available habitat in each year \\
\hline $\begin{array}{l}\text { Summary Habitat } \\
\text { Results }\end{array}$ & $\begin{array}{l}\text { Chart of available habit across the period of record or boxplot of the yearly values. Also used to } \\
\text { identify scenarios that deviate more than } 10 \text { percent from the baseline scenario }\end{array}$ \\
\hline $\begin{array}{l}\text { Flow Versus } \\
\text { Habitat Chart }\end{array}$ & Chart of the normalized habitat versus discharge functions \\
\hline Tabular Data & Spreadsheet of the data used to create any of the charts or non-spatial inputs \\
\hline
\end{tabular}

\section{Adding, Removing, and Resizing Modules}

New modules can be added to the application through use of the "Modules" menu. The selected module will appear with the default size and docking (right, left, top, or bottom). A module can be resized by hovering the cursor over one of the edges; when the resize icon appears, the user can click and drag the module to the desired size. A module can be moved by clicking and dragging the title bar of that module to a new position. While the module is being dragged, a set of docking icons will be visible as well as a ghost-blue image of the module's new location (figure 3). Modules can be removed by clicking on the $\mathrm{X}$ in the upper right corner. In addition to the basic docking locations within the application, users can also dock modules within other modules, drag modules outside the main application window, stack multiple modules onto one another, and pin modules so they auto hide when not in use. 


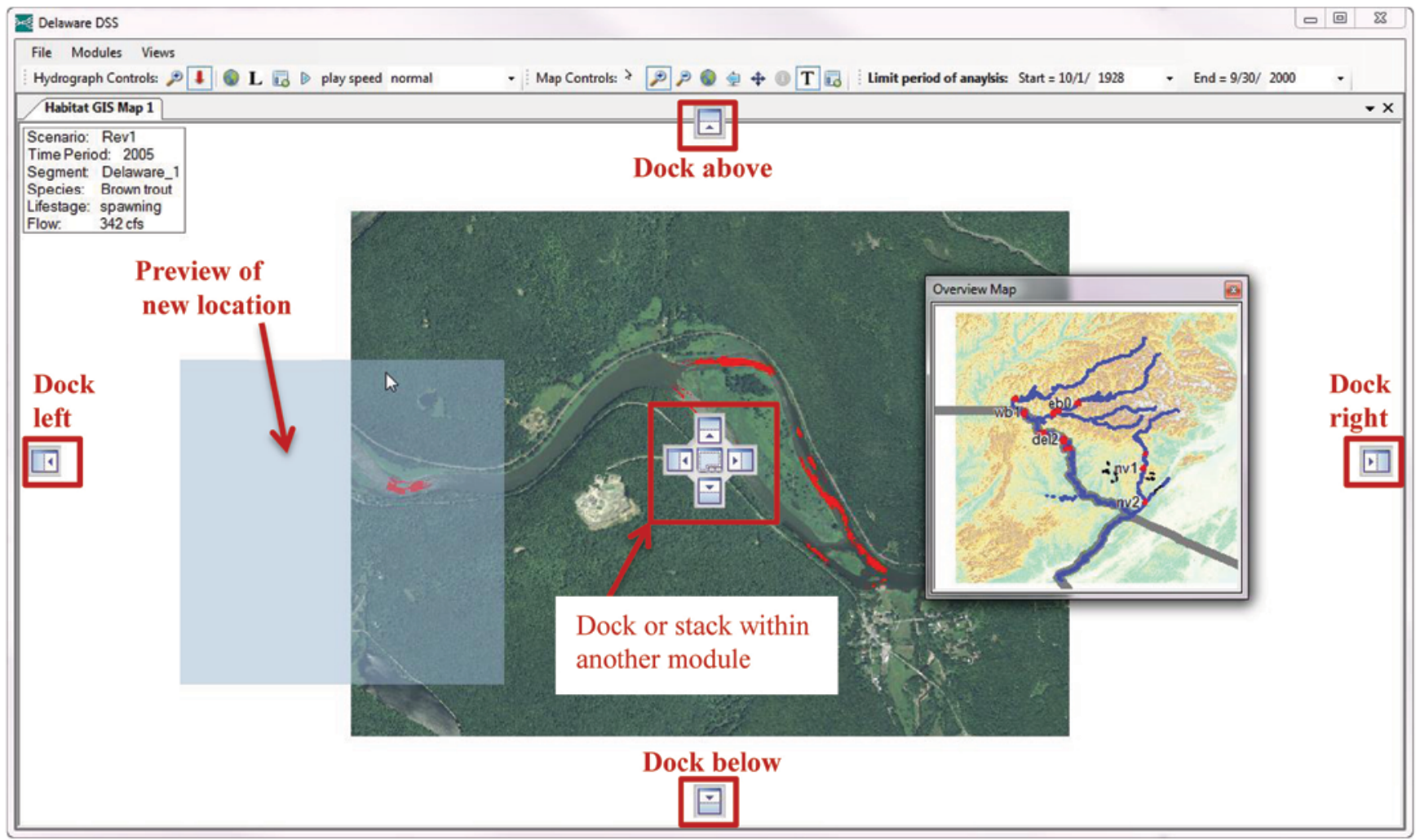

Figure 3. Module being moved and docked

\section{Using Views to Facilitate Organizing Multiple Modules}

The user will most often interact with multiple modules at once in the REFDSS. While it is possible to bring in and set up multiple modules individually, the REFDSS provides the ability to easily open, switch between, and save views. Version 1.1.2 of the REFDSS comes with several built-in views that users can select to open a specific module configuration. Built-in views (table 2) can be selected using the "Views" menu. User-defined custom views can be saved by clicking the "Views" menu and selecting "Save current view." The user will then be prompted to name the new view. "Remove view" is used for deleting a no longer needed view. Be careful when removing views as the view, even the builtin view, is permanently deleted. 
Table 2. Delaware REFDSS built-in module views

[DWM, dwarf wedgemussel; SF, shallow fast guild; SS, shallow slow guild]

\begin{tabular}{|c|c|}
\hline View & Description \\
\hline Brown Trout Summary & Summary habitat result modules for each brown trout life stage \\
\hline $\begin{array}{l}\text { Shad, DWM, SF, and SS } \\
\text { Summaries }\end{array}$ & Summary habitat result modules for remaining species \\
\hline Spatial Overview & A segment map module for each study section as well as an overview map module \\
\hline Main Stem Covariates & Summary of covariates on the main stem \\
\hline $\begin{array}{l}\text { Comparing } 2005 \text { to } 2010 \\
\text { Covariates }\end{array}$ & Comparison of 2005 and 2010 covariate inputs \\
\hline $\begin{array}{l}\text { Edit Habitat Suitability } \\
\text { Curves/Maps }\end{array}$ & View used to edit habitat suitability criteria and rerun geoprocessing \\
\hline System Wide Metrics & Display of the default system wide metrics \\
\hline Habitat Versus Discharge & View of flow versus Discharge module. Can be used to view any species \\
\hline $\begin{array}{l}\text { Brown Trout Adult Drill } \\
\text { Down }\end{array}$ & Demo showing how to trace back from a summary to the raw inputs \\
\hline $\begin{array}{l}\text { Comparing 1980s Versus } \\
\text { 1990s WB0 Brown Trout } \\
\text { Adult Habitat }\end{array}$ & Demo showing how to compare two time periods \\
\hline
\end{tabular}

\section{REFDSS Toolbars}

Three toolbars are docked at the top of the REFDSS application that can be used for interacting with various modules. The "Map Controls" toolbar is used to manipulate the map modules; the "Chart Controls" toolbar is used to manipulate the various output charts; and the "Limit period of analysis" toolbar changes the time period used for analyses.

\section{Map Controls Toolbar}

The Map Controls toolbar contains various items useful for interacting with the map modules (figure 4). The first four icons change the type of interaction and cursor the mouse has with the map. Users of online mapping or GIS will be familiar with these icons. The next two icons are used to either zoom to the full extent or the previous extent. The last icon will turn on or off the maps title box.

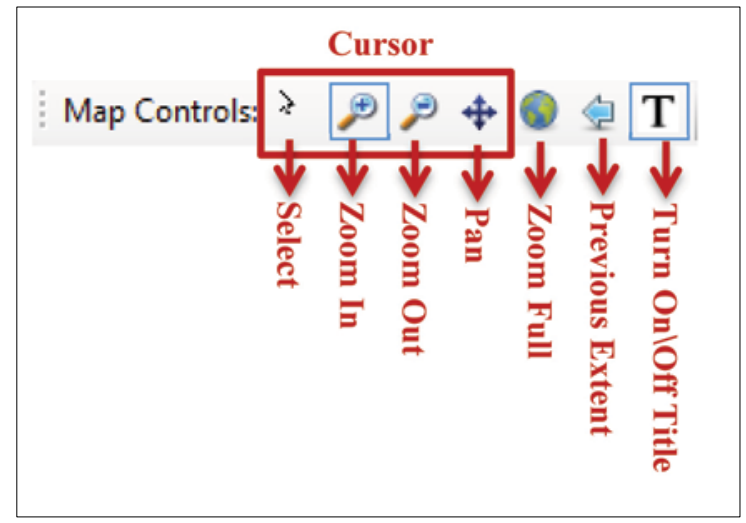

Figure 4. Map controls toolbar 


\section{Chart Controls Toolbar}

This toolbar contains items specific to the various chart modules (figure 5). The first two items change the type of interaction/cursor that the mouse has with charts. The "Zoom In" cursor is used to display a section of the chart in greater detail. This can be done by selecting the "Zoom In" button and then clicking and dragging over a section of a chart to make that subset of the data fill the chart area. The "Select" cursor is specialized to only work on the hydrograph chart modules. Clicking on a point in the hydrograph with the "Select" cursor will update a corresponding segment map based on the nearest representative flow used in the DSS (e.g., the flows used in 2-D hydrodynamic modeling, see Bovee and others, 2007, and Maloney and others, 2012). This can be used to determine how input covariates and output habitat change across a range of flows. The final two items on the Chart Controls toolbar allow the user to play a time-lapse animation of the map display showing the changes in response metrics to the hydrograph values. The view named "Edit habitat suitability curves/maps" is set up to provide a demonstration of this selection and animation. If the user opens this view and clicks on the hydrograph, the maps update to display layers corresponding to the selected flow. Clicking the "Play" button allows the user to view the animation; clicking "Play" again stops the animation.

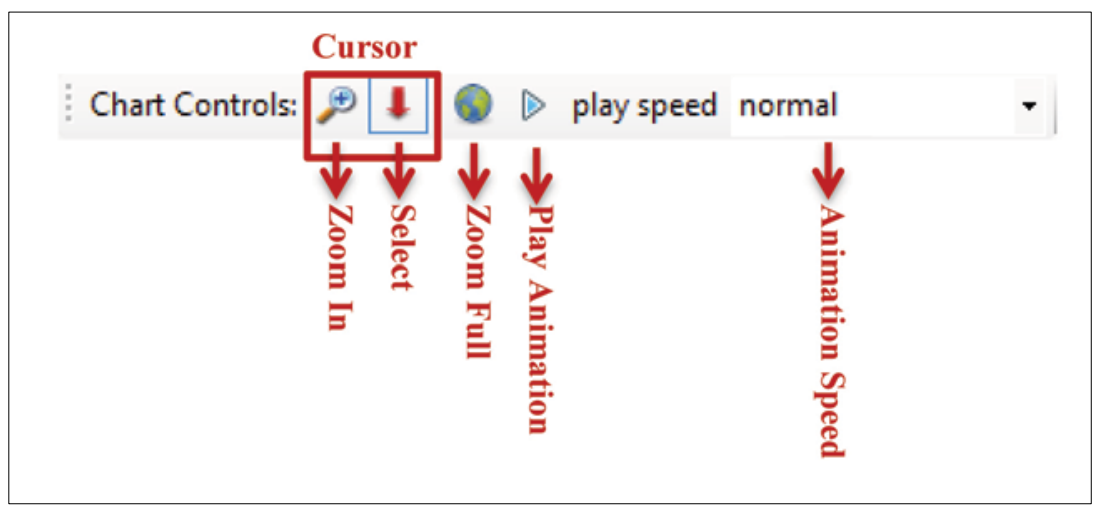

Figure 5. Chart controls toolbar

\section{Limit Period of Analysis Toolbar}

The final toolbar can be used to limit the entire period of record by selecting a start and an end year. Individual modules can have specific start and end dates that are more restrictive than the global values set here (see the "Comparing 1980s with 1990s" view for an example of this). Changing the values in this toolbar will update the values used in all current modules. By default, the entire period of record is 1929 through 2000 .

\section{REFDSS Modules}

\section{Overview Map}

The Overview Map module provides a means of viewing a map of the entire study area. Each of the 11 study segments are outlined in red (figure 6 shows 8 of the 11). The "Select" cursor in the Map Controls toolbar can be used to add a new segment map module by clicking on one of the red squares in the Overview Map module. 


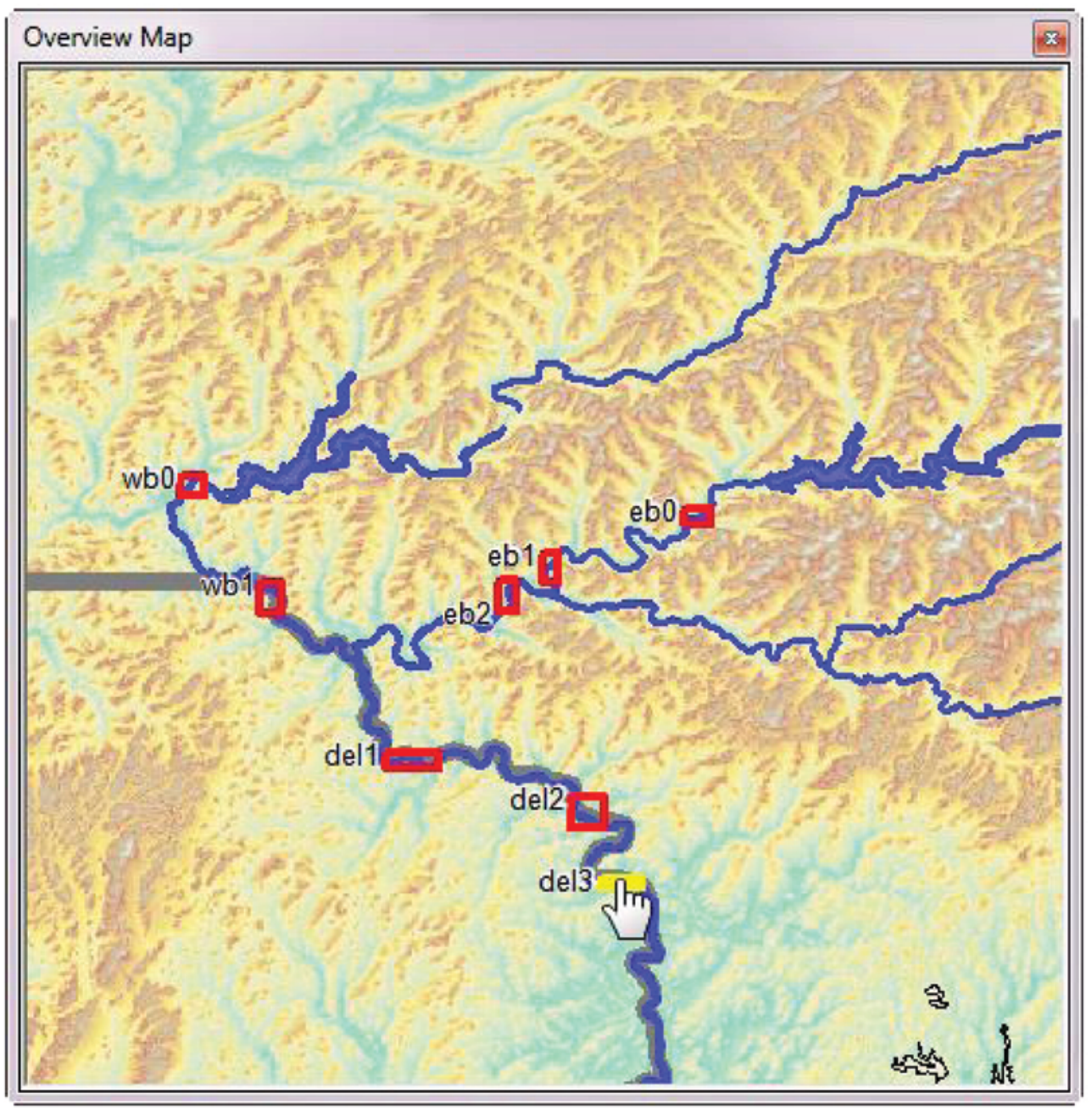

Figure 6. Overview map module

\section{Segment Map}

The Segment Map module provides a means of viewing a detailed map for an individual study segment. On top of an aerial image of the segment, the user can display one of the covariates or outputs. To change either the study segment or the overlaid map layer, right click on the map or its title bar and a context menu will appear. Hover your mouse over the scenario, segment, time period, species, life stage, covariate, or flow that you are interested in viewing. The list of available items under that category will appear (figure 7). Note: Not all species are available for all segments or time periods. For example, data for DWM are only available for 2010 and for segments Del1, Del2, and Del3. To display or remove the title box, click the "T" icon on the map toolbar. 


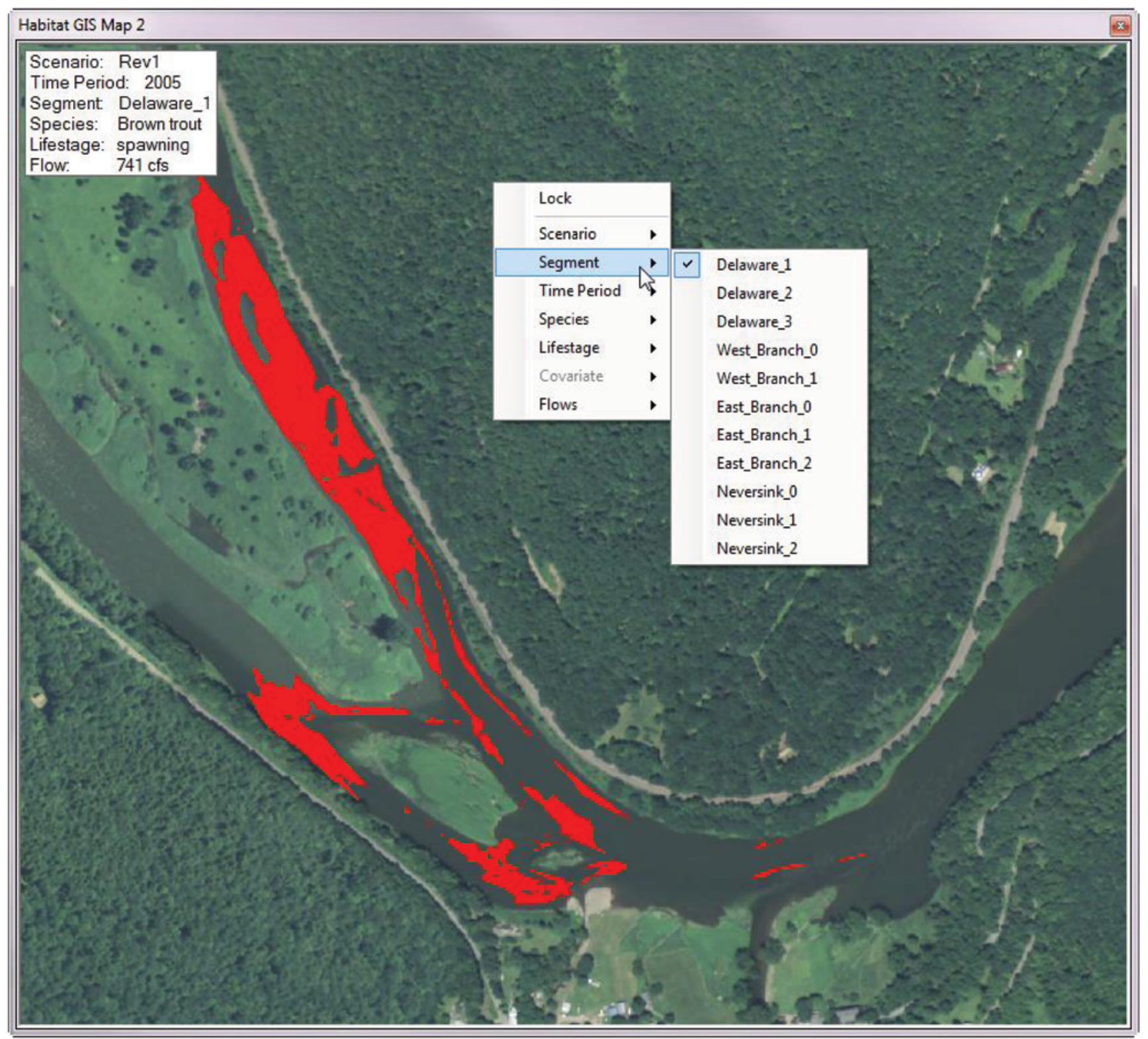

Figure 7. Segment map module

\section{Habitat Suitability Generator}

The Habitat Suitability Generator module provides functionality to view and change the HSC used to generate habitat maps for the REFDSS. Additionally, it allows the user to view and edit the equation used to generate the habitat maps. It also contains functions to regenerate the map outputs when changes to the HSC or equations have been made (figure 8). For this version of the REFDSS (v1.1), HSC for fishes were taken from Bovee and others (2007) and HSC for the DWM were taken from Maloney and others (2012). These HSC are currently undergoing literature verification and may be modified in the future as more data become available. 


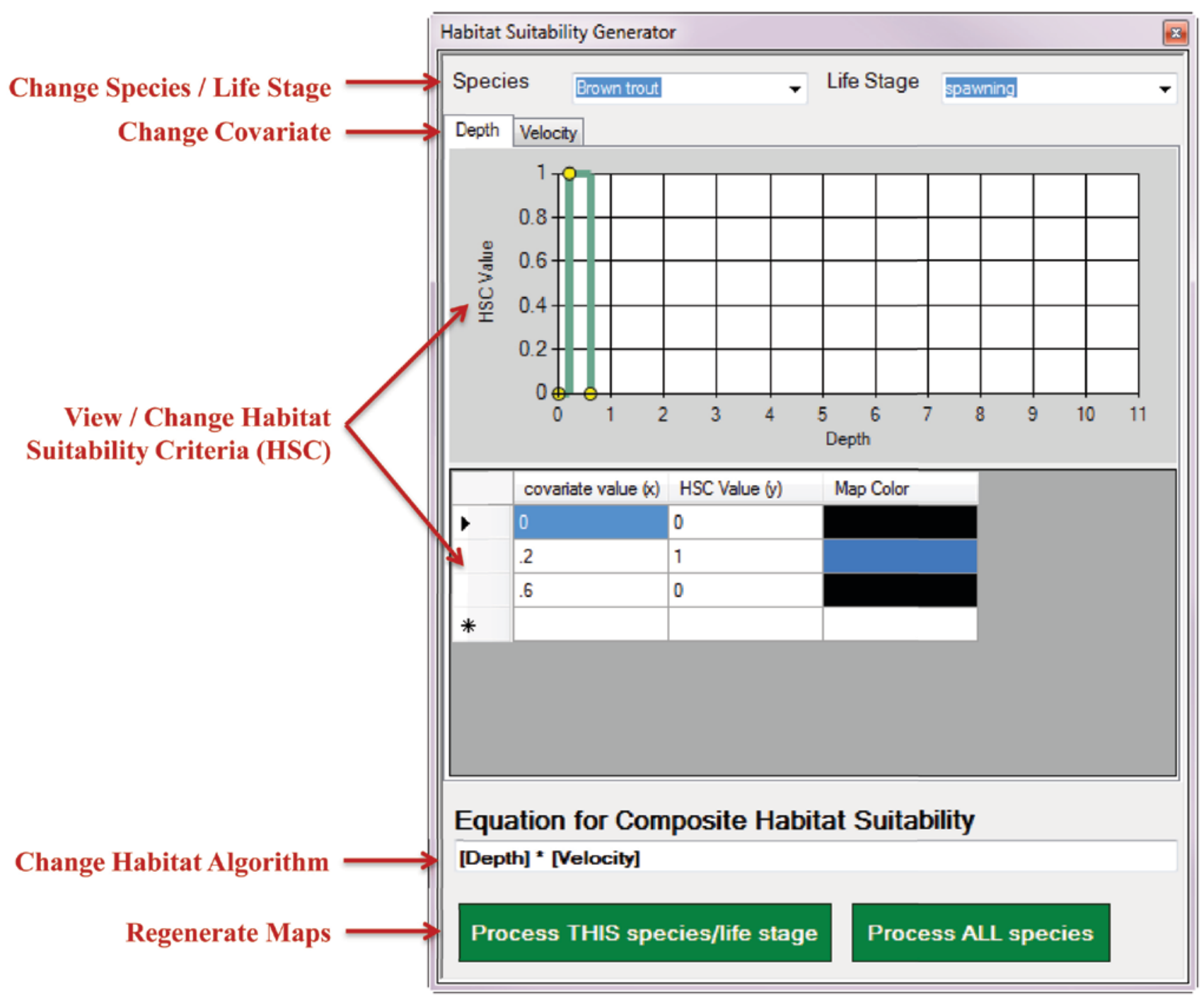

Figure 8. Habitat suitability generator module

In the center of this module, there is a chart that displays an HSC curve for a single species, life stage, and covariate. Beneath that curve is a list of its $\mathrm{x}$ and $\mathrm{y}$ values. Edits to the curve can be made by either changing the values in the table or clicking and dragging on one of the yellow dots on the curve chart. The drop-down boxes at the top of the module can be used to change the species or life stage HSC displayed. To switch between different input covariates (e.g., depth and velocity), select the tabs immediately above the chart. Any changes to the HSC are automatically saved, replacing the original values. To undo these changes and revert to the original HSC values, click "File" and select the “Reset all Defaults!" option.

If a segment map module is visible, any changes to the HSC will be reflected in the spatial habitat inputs. To try this, open the "Edit habitat suitability curves/maps" view. Change one of the HSC values in either the chart or table to get the segment map modules to update their display. The two segment map modules are displaying depth and velocity using the default symbology for those layers. The third column in the table below the HSC chart shows the color that will appear on the map for that 
bin of the HSC. Double click on the colored box to change this color. Black indicates areas that will be displayed as transparent.

Once all changes to the HSC have been made, the user can update the equation used in the text box entitled "Equation for Composite Habitat Suitability." Standard mathematical symbols can be used with the covariates contained in square brackets. For example, the default algorithm for brown trout spawning (after Bovee and others, 2007) is "[Depth] * [Velocity]." This means that the depth grid (or map) will be reclassified according to the depth HSC and then multiplied by the velocity grid and reclassified according to the velocity HSC. If the user wanted to change this algorithm to be the square root of (depth squared * velocity squared), for example, the equation would be entered as "([Depth $]^{\wedge} 2 *$ $\left.\left.[\text { Velocity }]^{\wedge} 2\right)^{\wedge} 0.5\right)^{\prime}$.

Once the equations have been set, the outputs can be regenerated. To process the outputs for a single species/life stage, click the "Process THIS species/life stage" button. The processing time for a single species depends on the computer's speed and memory but should be around 20 minutes. To reprocess all of the species and life stages, click the "Process ALL species" button. The processing time for all species takes considerably longer, somewhere around four hours, as of the time of the writing of this publication.

\section{Chart Output Modules}

These modules, which include Hydrograph, Yearly Habitat Results, Summary Habitat Results, Sytem Wide Metrics and Flow Versus Habitat Chart, are used to display various outputs in a graphical or chart-based form. Each module has a context menu that can be opened by right clicking in the module or its title bar. The context menu lists several useful options including printing the chart, saving a .jpeg image of the chart, or copying it to the clipboard (for use in another application, for example).

\section{Change the Displayed Data in a Single Chart}

The context menu (obtained by right clicking on a chart) contains an option titled "Select Data" which allows the user to change the data that the chart is displaying. Selecting this opens an interface that allows the user to select multiple items for display. Note that this is not implemented for the System Wide Metrics chart because of its unique data display (see System Wide Metrics subheading below for more information). This interface will be slightly different for each of the output chart modules (figure 9). For example, the scenario and baseline selection box will not be visible in a "Flow Versus Habitat Chart" because they are the same for all scenarios. When multiple items are selected in each of the categories, the total number of items displayed in the resulting chart is multiplicative. For example, when 3 scenarios, 2 time periods, 4 river/segments, and 6 species/life stages are selected, the resulting output chart will contain 144 elements. This can quickly lead to indecipherable charts, so it is best to limit the cumulative number of elements in any given chart and instead have multiple charts, each with a specific item of interest. 


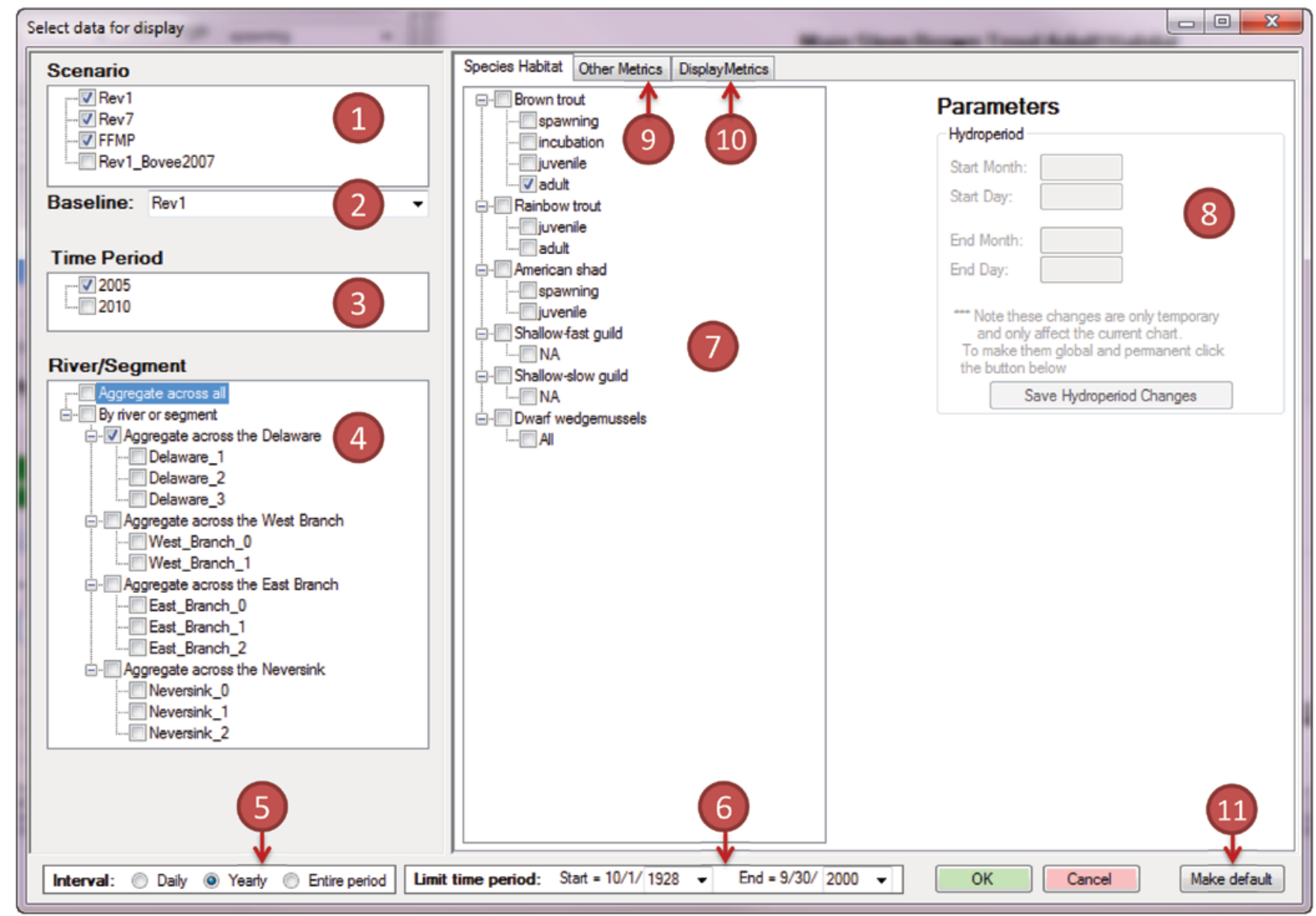

Figure 9. Select data window

While there are numerous elements on the "Select Data window," their use is relatively straightforward. Note that not all options will be available for selecting data for all charts.

The options are:

1. Scenario-Select which of the available scenarios to compare.

2. Baseline - Select one of the flow scenarios to be used as a baseline to compare against the remaining flow scenarios. This is used in the "Summary Habitat Chart" and the "Tabular Data" modules to measure percent change. Changes greater or less than $10 \%$ are flagged in green and red respectively.

3. Time Period-Select which of the available time Periods to display.

4. River/Segment - Select which river or study segments will be displayed. Results for individual segments can be displayed by checking the innermost nodes (e.g., Delaware_1). Metrics can be summarized at the branch scale (e.g., for an individual river) by selecting the "Aggregate across the ..." nodes immediately above the individual segment nodes. The method used to summarize or aggregate results across a river is described in detail in Bovee and others (2007, p. 31). The "Aggregate across all" option provides the sum of the totals for each river reach summarization. Note that certain charts do not support the aggregation of data. Finally, data summarization is 
done dynamically for all charts as they are being updated-aggregating across rivers or across the Basin may take some time, especially when using the full time period of record.

5. Interval-Select a method of temporal aggregation of the results. "Daily" shows the raw data and is only used in the "Hydrograph" and the "Tabular Data" modules. The "Yearly" option will calculate a given metric for each individual year. This allows users to observe temporal variability and changes over time. This option is currently only available in the "Summary Habitat Results" and the "Tabular Data" modules, although the "Yearly Habitat Results" uses it by default as well. The final option is "Entire period" which does not aggregate results by year but summarizes them over the entire hydroperiod. This option is the same as that used in the original Bovee and others (2007) DSS.

6. Limit time period - Used to limit the time period of a single chart or tabular data output. To globally change the period of record, use the "Limit period of analysis" toolbar at the top of the main application form.

7. Species Habitat - Specify which species/life stages will be displayed. The top level check boxes next to each species do not have an effect; there is not currently a way of summarizing across all life stages for a given species.

8. Parameters (for species habitat) —With a life stage selected, the hydroperiod parameters are enabled and display the values used for that life stage. The values in the hydroperiod boxes can be changed, but changes are temporary and only apply to the single module linked to this "Select Data" window. To make changes permanent and global, click the "Save Hydroperiod Changes" button.

9. Other Metrics - Select and display non-habitat metrics such as storage volumes, releases, diversions, targets, and spills. Note that this is only available for the "Hydrograph" or the "Tabular data" modules.

10. Display Metrics - Change the aggregation algorithm used to calculate the yearly and entire hydroperiod summaries. The default value "Average of bottom 25 percent of habitat" is equivalent to what was used in the original DSS. In addition, "Minimum single habitat" in which the available habitat from the time step with the least area is reported and "Average all Habitat" in which the available habitat is averaged across all time steps in the hydroperiod are available.

11. Make Default - Save the current selections to be the default for all new modules of this type.

\section{Change Individual Series Symbology and Label}

Adjustments can be made to the color, line width, line type, and displayed title for improved chart readability. To do this, click on the series symbol in the chart legend to bring up a simple interface for changing these attributes (figure 10). These changes are saved globally so the next time users bring this series into a new chart it will have this label and symbology. 


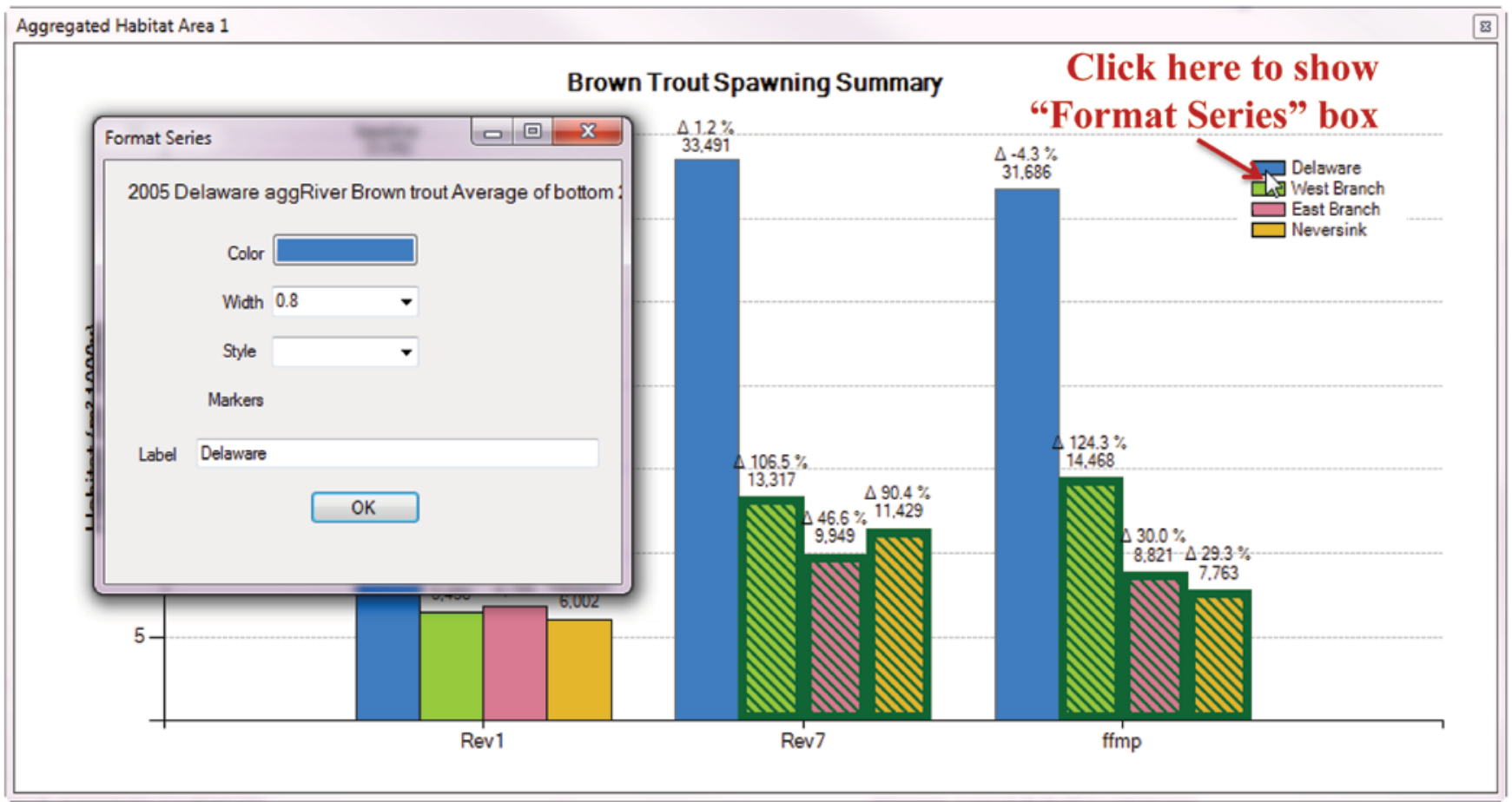

Figure 10. Format series

\section{Format a Chart}

To change the default chart display settings, right click on a chart and select "Format Chart." A window will appear that allows users to change the following elements: title text, title font, title location, axis labels, axis font, axis scale, tick marks, and grid lines. Much like the Select Data window, changes can be saved as the default for this chart type using the "Make default" option.

\section{Hydrograph Module}

The "Hydrograph" module can be used to display a line graph of daily flow (Q), habitat, or any of the other metrics. Flow series are depicted on the left or primary Y-axis while other daily series are represented on the right or secondary Y-axis (figure 11). Note that when displaying multiple disparate metrics, they might not be readable on a single scale. For example, it is not possible to have a chart that meaningfully shows drought conditions (values $0-3$ ) at the same time as showing Beaverkill smoothed $\mathrm{Q}$ (values $0-17,000$ ). However, hovering the mouse pointer over any line will display a small box containing the exact date and value at that point.

To zoom in on a section of the chart, click the zoom icon on the Chart Controls toolbar and click and drag a box around a section of interest. When zoomed in, users can scroll using the scroll bar at the bottom or left of the chart. To zoom back out, either double click the global extent icon on the Chart Toolbar, one of the small circles at the left end of the X-axis scroll bar or the top of the Y-axis scroll bar.

When displaying habitat areas on the hydrograph chart, only data for the time periods corresponding to the hydroperiod will be shown. These temporal extents will be highlighted with 
colored boxes on the chart. If a segment map is displayed and set to the same flow scenario depicted on the hydrograph, the map will sync with the selected flow. To try this, open the "Edit habitat suitability curves/maps" view. Click Select cursor on the Chart Controls toolbar and click on the hydrograph. All three maps will update to display the habitat or covariate data for the flow that most closely matches that selected on the hydrograph. An animation of these flows over time can be viewed by clicking the "play" button on the Chart Controls toolbar.

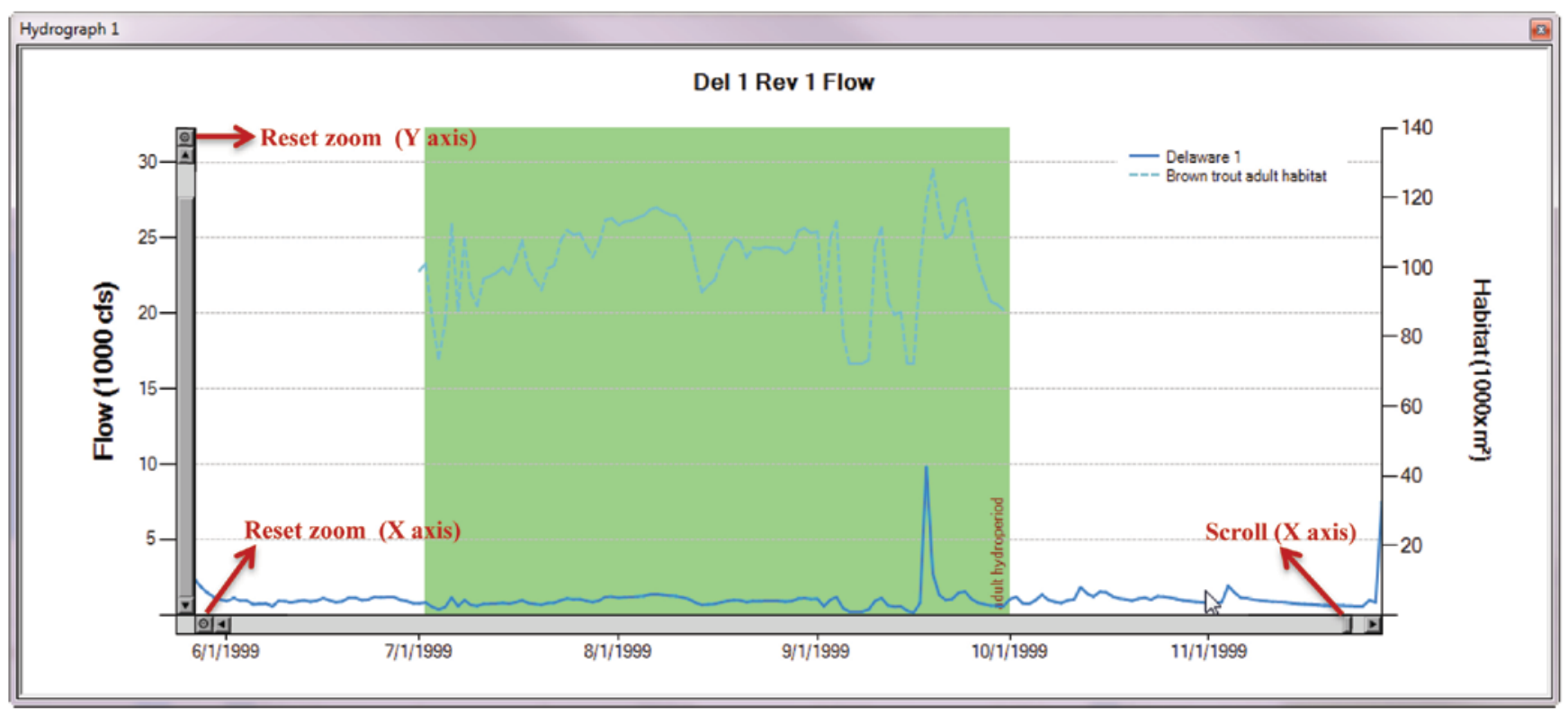

Figure 11. Hydrograph chart

\section{System Wide Metrics}

The "System Wide Metrics" chart (figure 12) is used to view a summary of several non-habitat metrics including "Montague shortage magnitude Major \% of Minimum delivery," "NYC shortage magnitude Major \% of Minimum delivery," "New Jersey shortage magnitude Major \% of Minimum delivery," and "Drought days.", which are described in Bovee and others (2007, p. 29). Unlike the other charts, switching between metrics and changing the parameters for System Wide Metrics charts is done through a context menu displayed by right clicking on the chart. The "Metric" item in this menu allows the user to select one of the four metrics while the "Parameters" item allows the user to change the parameters used to calculate the value. 


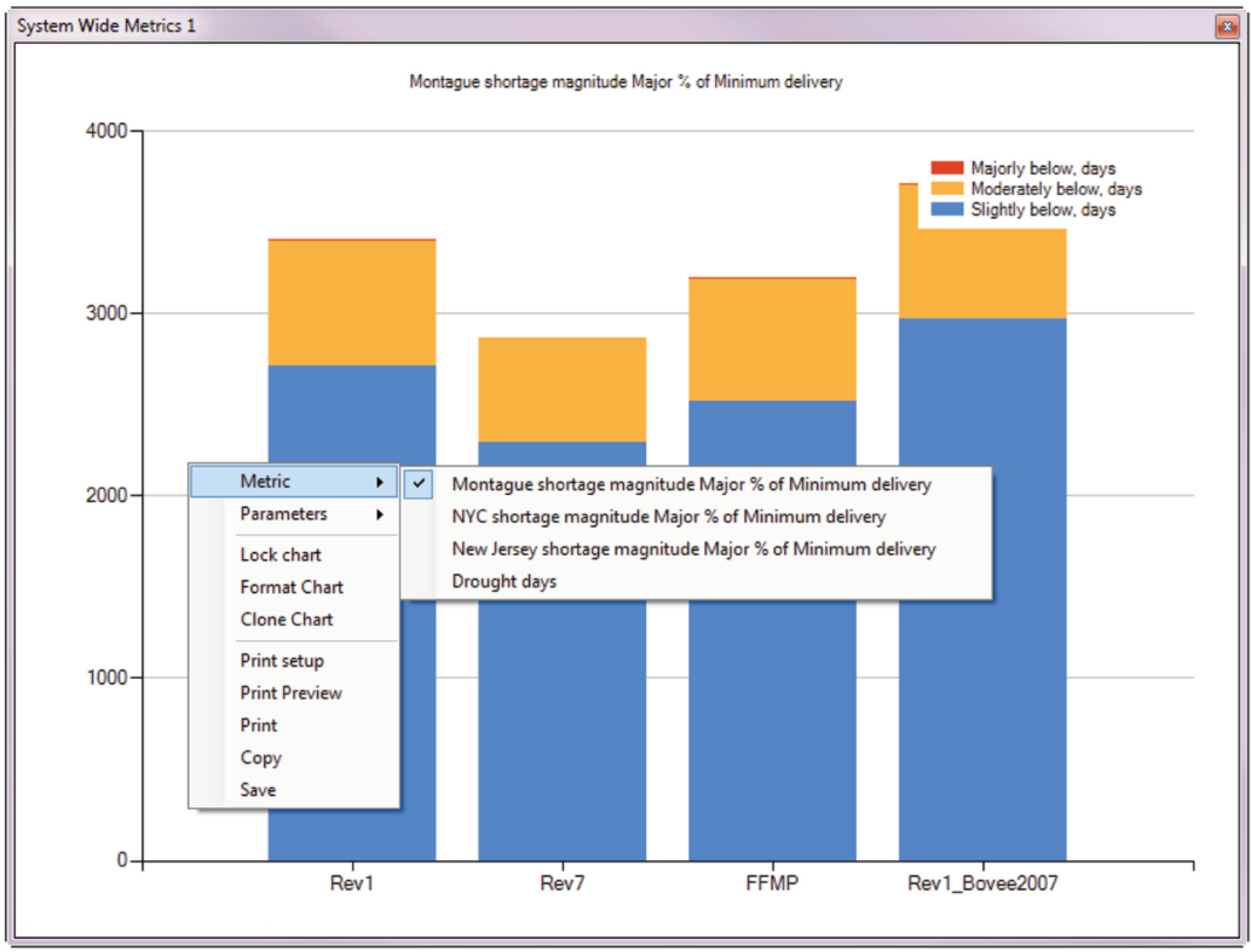

Figure 12. System wide metrics

\section{Yearly Habitat Results}

The "Yearly Habitat Results" module is used to view all the habitat results summarized by year (figure 13). This is similar to the daily habitat for a species in a hydrograph chart; instead of a line graph, data are depicted in a bar chart for each year. Zooming and panning a "Yearly Habitat Results" chart will simultaneously sync the extent of any open hydrographs. This allows users to pan through the period of record simultaneously to determine the single events that led to the yearly observations. This interaction can be seen in the bottom two charts in the "Brown Trout Adult Drill Down" view. 


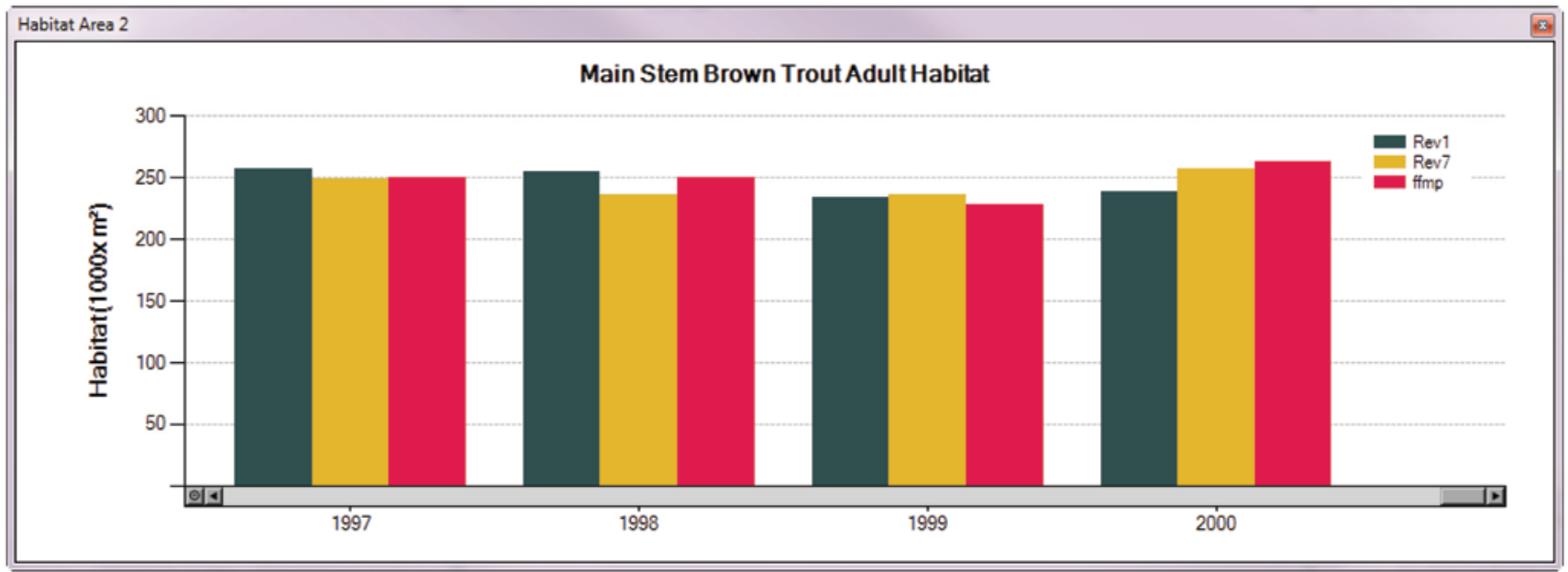

Figure 13. Yearly habitat chart

\section{Summary Habitat Results}

The "Summary Habitat Results" module presents a bar chart or boxplot of habitat area for a given species/life stage. The Summary Habitat Results chart can display output in two distinct ways. If users select "Entire Period" as the interval (under Select Data from the context menu), a single value will be displayed for each scenario. This value takes the daily values for the entire period of record and applies the selected display metric (by default "Average of bottom $25 \%$ of habitat") to the full list at once. This is how these values were calculated in the original DSS. If users select an interval of "Yearly" instead, the display metric is a boxplot of each year's data (figure 14). The points displayed in the boxplot are the minimum, maximum, $25^{\text {th }}$ and $75^{\text {th }}$ percentiles, mean, and median.

The Summary Habitat Results chart allows a user to select a baseline scenario to compare the other scenarios against (using the Select Data option from the context menu). The percent change between this baseline and each chart is displayed above each bar on the chart. If one scenario performs 10 percent or more above the baseline, its bar is highlighted in green (figure 15); a decrease of 10 percent or more from baseline will be highlighted in red. 


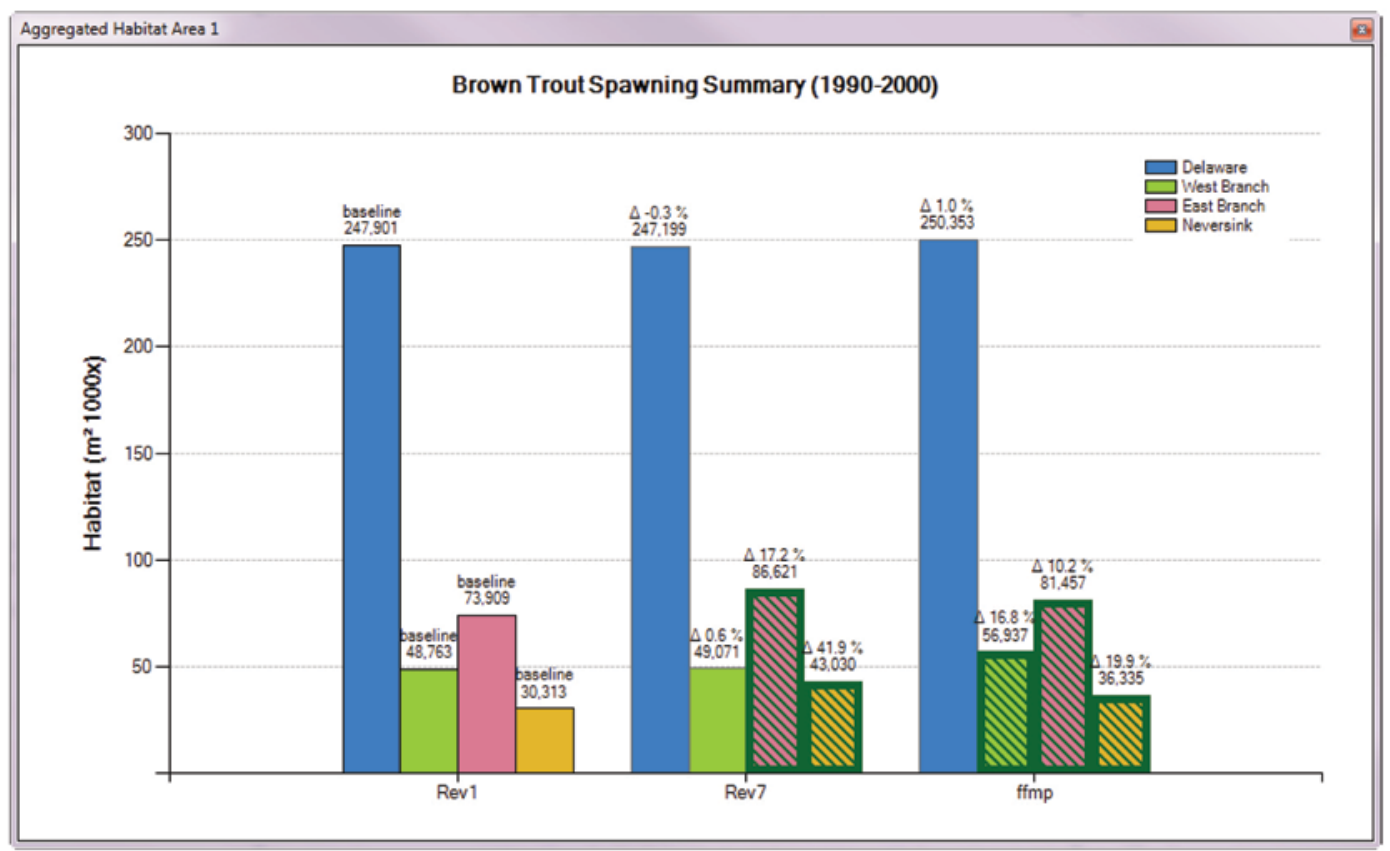

Figure 14. Summary habitat chart with yearly option

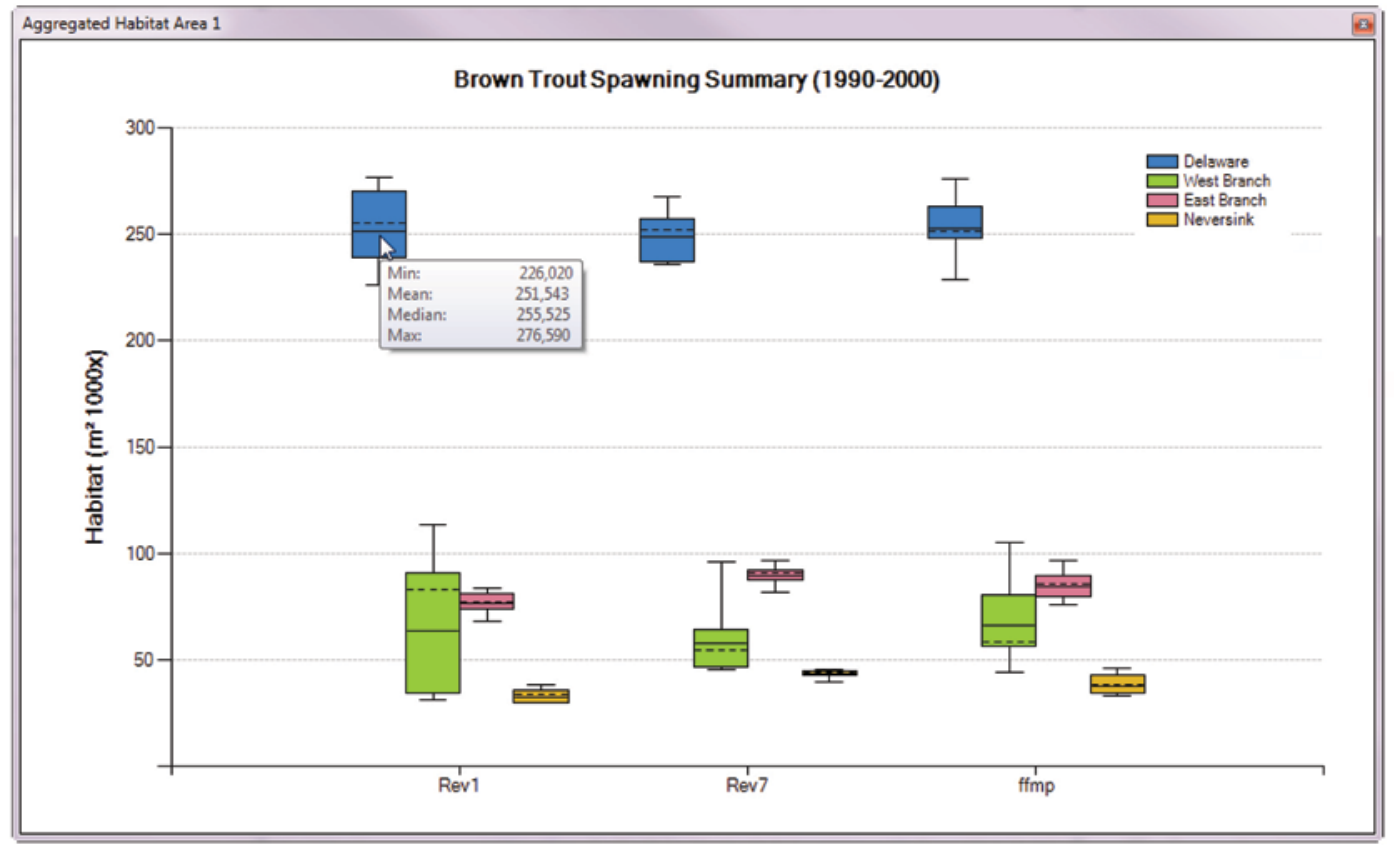

Figure 15. Summary habitat chart with entire period option 


\section{Flow Versus Habitat Chart}

The "Flow Versus Habitat Chart" module presents a means of visualizing the habitat area as a function of discharge (figure 16). The techniques used to generate these charts are described in Bovee and others (2007, p. 37).

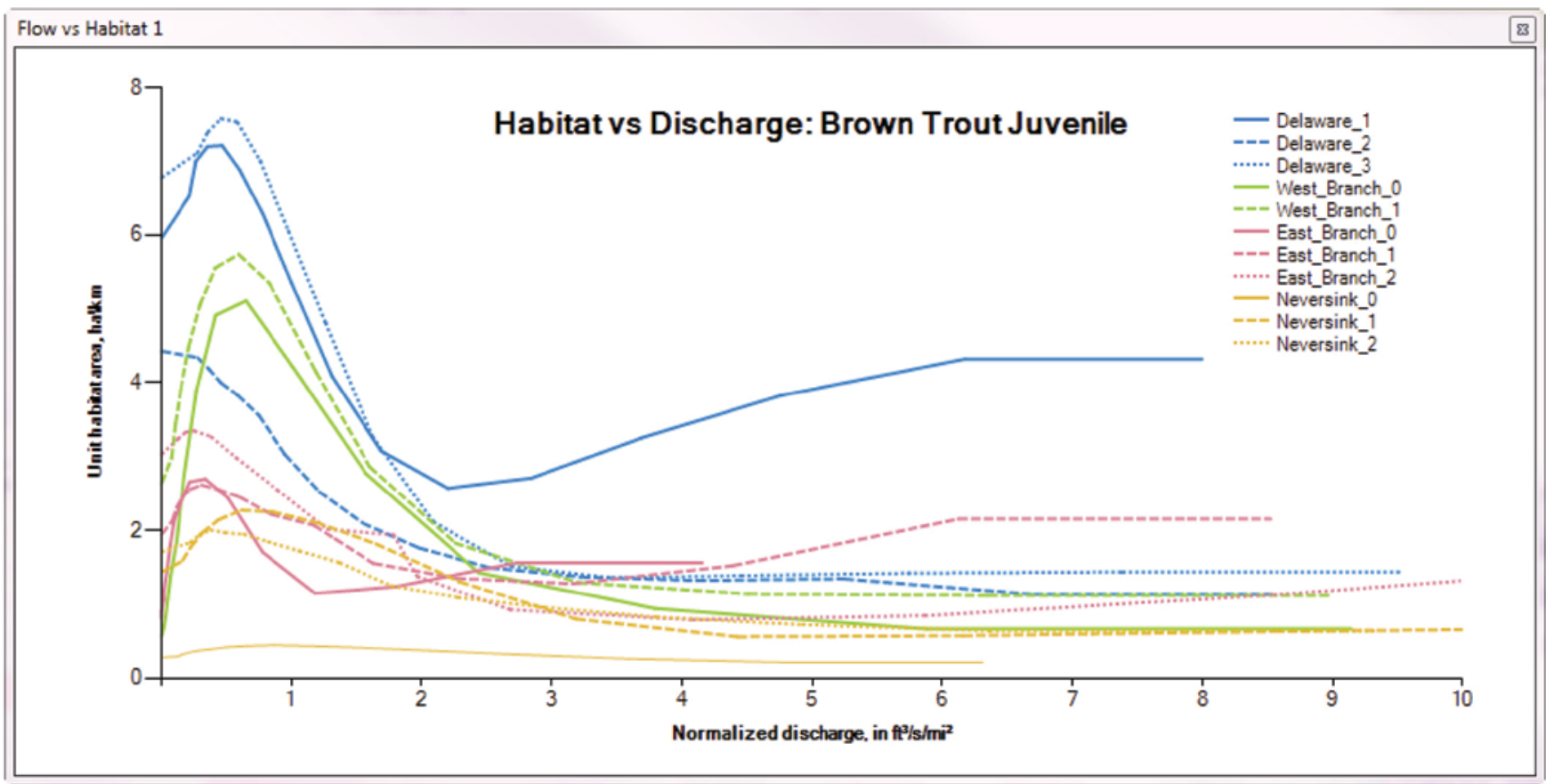

Figure 16. Flow habitat chart

\section{Tabular Data Module}

Each of the charts described above relies on a series of values extracted from a simple SQLite database using complex SQL queries. The Tabular Data module facilitates direct access to the raw data inputs used in individual charts. By opening the Select Data window (from the table's context menu), the user can select which parameters are displayed in tabular format. Additionally, many of the charts will directly allow users to view their corresponding raw data values by selecting the "View Tabular Data" option from the chart's context menu.

The context menu for the Tabular Data module also contains options for copying the currently selected data or all data to the clipboard. Once on the clipboard, the data can be directly pasted into Excel. Additionally, there is an option to save all data to an external text file in comma-separated value (CSV) format. This saved file can then be brought directly into most other data analysis and visualization programs including R, Python, Excel, or Microsoft Access.

The "Tabular Data" module uses the same red and green color coding of deviations from the baseline as in the "Summary Habitat Results" chart. This only displays when "Yearly" or "Entire period of record" is selected as the interval. 


\section{Limitations and Future Work}

This version of the REFDSS platform aims to recreate the functionality of the original Bovee and others (2007) — DSS in a more user friendly and transparent manner. The output produced by this software has been cross-checked with the original output to verify that both are producing similar values. Due to underlying computational differences employed between the two versions (vector versus raster GIS processing, for example), the two outputs will vary slightly. Two items from the original DSS (temperature thresholds and spawning/incubation persistent habitat) were not included in this version. These items are being actively developed and will be included in a future release of the REFDSS application. Additional items currently being researched include using inputs created with bathymetric lidar to extend the study segment extents and using a dynamic metric of persistence that accounts for fluctuations in habitat for species with limited mobility.

\section{References Cited}

Bovee, K.D., Waddle, T.J., Bartholow, J., and Burris, L., 2007, A decision support framework for water management in the upper Delaware River: U.S. Geological Survey Open-File Report 2007-1172, $122 \mathrm{p}$.

Maloney, K.O., Lellis, W.A., Bennett, R.M., and Waddle, T.J., 2012, Habitat persistence for sedentary organisms in managed rivers: the case for the federally endangered dwarf wedgemussel

(Alasmidonta heterodon) in the Delaware River: Freshwater Biology, v. 57, p. 1315-1327. 


\section{Appendix 1-Credit for Open-Source Components Used}

The development of the Delaware REFDSS would not have been possible without the use of several open-source and free projects.

- GIS map display is provided by the MapWinGIS ActiveX Control Project which is part of the MapWindow GIS Open Source Project (http://www.mapwindow.org/)

- The user configurable docking windows are from the DockPanel suite available at http://dockpanelsuite.sourceforge.net/

- The database back end uses SQLite with the dot.net bindings (http://www.sqlite.org/about.html)

- Unzipping functionality uses the DotNetZip Library (http://dotnetzip.codeplex.com/)

- Charting functionality was through the built-in Microsoft Charting Library 